\title{
Belgeo
}

Revue belge de géographie

$1 \mid 2014$

From urban renewal to metropolitan strategies ?

Cultural flagship projects in restructuring industrial areas

\section{La reconversion de l'aire industrielle de Bagnoli à Naples : anatomie d'un échec}

The conversion of the industrial area of Bagnoli in Naples: failure's anatomy

\section{Elodie Manceau}

\section{OpenEdition}

\section{Journals}

Édition électronique

URL : http://journals.openedition.org/belgeo/12718

DOI : 10.4000/belgeo.12718

ISSN : 2294-9135

Éditeur :

National Committee of Geography of Belgium, Société Royale Belge de Géographie

\section{Référence électronique}

Elodie Manceau, «La reconversion de l'aire industrielle de Bagnoli à Naples : anatomie d'un échec », Belgeo [En ligne], 1 | 2014, mis en ligne le 15 décembre 2014, consulté le 01 mai 2019. URL : http:// journals.openedition.org/belgeo/12718; DOI : 10.4000/belgeo.12718

Ce document a été généré automatiquement le 1 mai 2019.

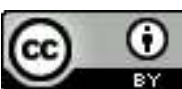

Belgeo est mis à disposition selon les termes de la licence Creative Commons Attribution 4.0 International. 


\title{
La reconversion de l'aire industrielle de Bagnoli à Naples : anatomie d'un échec
}

The conversion of the industrial area of Bagnoli in Naples: failure's anatomy

\author{
Elodie Manceau
}

\section{Introduction}

1 Les grands projets d'équipements culturels fondés sur l'économie de la connaissance, la durabilité et l'innovation illustrent les politiques publiques engagées par les villes anciennement industrialisées telles qu'Essen, Bilbao, Gènes, Belval et d'autres depuis le milieu des années 1990. Par exemple, à Bordeaux, la reconquête des quais de la rive droite à travers la libération des friches industrielles et militaires permet à la fois un recentrage de la ville centre et offre des opportunités foncières en matière de logements, d'équipement, de poumons verts ou encore d'espaces récréatifs. De ce point de vue, Bagnoli, situé dans la partie occidentale de Naples, pourrait être une formidable opportunité, mais va se révéler non seulement un échec assez singulier et bien au-delà, un exemple révélateur, à nouveau, des faillites quasiment permanentes des politiques de développement urbain dans les villes de l'Italie du sud et tout particulièrement à Naples.

2 L'attention et le succès de la Troisième Italie (Bagnasco, 1977 ; Trigilia, 1986 ; Pecqueur et Courlet, 1991) depuis la fin des années 1970 ont pu faire oublier un moment la permanence de la seconde Italie : cette Italie du sud et Naples au premier plan. Au-delà des discours et des programmes lénifiants, de modèles largement repris des thématiques européennes, le Sud italien reste perçu comme une formidable «machine » à aspirer des fonds qui généralement deviennent des fonds perdus pour les opérations envisagées, programmées avec d'autant plus de soins et de précisions qu'on sait qu'elles ne seront parfois pas réalisées. A nouveau, « le syndrome du Guépard » demeure le modèle dominant et traduit bien les comportements d'accaparement de certains acteurs. Dans Le guépard, encore, on sait que l'habillage, les « costumes » jouent un grand rôle dont personne n'est finalement dupe... 
3 Le projet de reconversion industrielle de Bagnoli s'inscrit aussi dans ce mouvement général identifié aujourd'hui sous la notion de "smart city » et accentué cette dernière décennie par le processus de métropolisation, entendu aussi bien au sens géographique reconstruire la ville et la centralité sur la ville - qu'au sens économique visant la concentration des fonctions de commandement, de coordination, de contrôle, de création de codes (Lacour et Puissant, 1996 et Bourdeau-Lepage, 2012).

4 Fleuron de la sidérurgie nationale jusque dans les années 1980, le site de Bagnoli a fermé ses portes en 1993 sous les injonctions de la Communauté européenne. Cette friche de 330 hectares est au centre de controverses et reflète les nombreux dysfonctionnements, les conflits et les blocages rencontrés par les acteurs institutionnels. Les financements attribués se comptent par millions d'euros et les résultats sur le terrain restent peu visibles aux yeux de la presse, de nombreux responsables politiques et des citoyens. L'incendie en 2013 du musée de la Città della Scienza ${ }^{1}$, symbole du renouveau du site, la mise en examen de 21 ex-dirigeants de la société de transformation BagnoliFutura ${ }^{2}$ pour pollution aggravée des terrains de l'ancienne aciérie et la mise en faillite de la même société à l'été 2014 ravivent et illustrent des difficultés toujours présentes auxquelles doit faire face le territoire. Or le site rencontre aussi un fort problème d'image lié entre autres à la présence supposée de la mafia napolitaine, dans des affaires de marchés publics et de bonification des terrains ${ }^{3}$.

5 C'est donc par le biais de projets d'aménagement d'envergures sur des espaces périurbains ou même centraux longtemps restés en marge que la plupart des grandes villes prétendant être ou devenir métropoles se positionne pour s'affirmer sur le plan national, européen, voire international dans un contexte de compétition accrue des territoires: mais quelle est la véritable ambition de Naples sinon être d'abord napolitaine ? Si le site de Bagnoli concentre les composants nécessaires à un processus de transformation urbaine positive, tenant à une situation géographique favorable, un paysage d'exception (les Champs Phlégréens), il canalise, exacerbe, catalyse aussi un certain nombre de difficultés : lenteur des procédures, immobilismes institutionnels et politiques, représentations négatives: une idiosyncrasie culturelle profonde, alimentée paradoxalement par l'abondance de financement, une coupable myopie de nombreuses institutions sur des comportements pour le moins douteux, pour ne pas dire coupables ou même mafieux.

Notre lecture, à défaut de pouvoir connaitre les mécanismes et les comportements réels privilégiera les jeux d'acteurs institutionnels et de leur articulation, des représentations et images liées au projet et au territoire dans lequel il s'inscrit.

\section{Bagnoli : une reconversion au sort incertain dans une métropole napolitaine malade}

7 La reconversion du site de Bagnoli s'inscrit dans un contexte territorial peu favorable au processus de métropolisation recherché par Naples, qui se revendique laboratoire de choix dans la mise en œuvre des politiques publiques locales et la recomposition des jeux d'acteurs «épurés» à l'image de la "renaissance napolitaine ${ }^{4}$ impulsée par la Municipalité d'Antonio Bassolino au milieu des années 1990. 


\section{Naples. Une métropole en décrochement persistant}

8 Naples est une métropole en décrochement persistant. Si elle appartient aux «grandes villes à potentiel européen » au même titre que Turin ou Bologne comme le suggère l'étude de $\mathrm{C}$. Rozenblat et $\mathrm{P}$. Cicille sur l'analyse comparée des villes européennes à partir de 15 indicateurs de fonctions métropolitaines (transports, universités, culture...) (Rozenblat, Cicille, 2003), elle reste néanmoins une métropole de plus de trois millions d'habitants, avec une forte densité dont près d'un million d'habitants pour la seule ville de Naples déclassée par son faible poids économique (Delpirou, Rivière, 2013; Manceau, 2011). Elle se classe, par sa population, parmi les trois premières métropoles d'Italie (Tableau 1).

Tableau 1. Approche comparée des métropoles italiennes. Quelques indicateurs au niveau provincial.

\begin{tabular}{|l|l|l|l|l|l|}
\hline Métropoles & $\begin{array}{l}\text { Population } \\
\text { (Istat, 2012) }\end{array}$ & $\begin{array}{l}\text { Population } \\
\text { Commune } \\
\text { centre (Istat, } \\
\text { 2012) }\end{array}$ & $\begin{array}{l}\text { Taux de (Istat, } \\
\text { chômage en } \\
\text { 2013) }\end{array}$ & $\begin{array}{l}\text { Densité de } \\
\text { population } \\
\text { (Istat, 2012) }\end{array}$ & $\begin{array}{l}\text { PIB par habitant } \\
\text { de 2010 (\% moyenne } \\
\text { UE27=100 } \\
\text { (Eurostat) }\end{array}$ \\
\hline Rome & 4039813 & 2638842 & 11,3 & 750,7 & 128 \\
\hline Milan & 3075083 & 1262101 & 7,7 & 1946,5 & 179 \\
\hline Naples & 3055339 & 959052 & 25,7 & 2607,6 & 65 \\
\hline Turin & 2254720 & 872091 & 11,4 & 330 & 113 \\
\hline Bari & 1246297 & 313213 & 19,9 & 325,8 & 78 \\
\hline Bologne & 990681 & 380635 & 8,4 & 267,5 & 141 \\
\hline Venise & 847983 & 259263 & 8,6 & 343,8 & 112 \\
\hline Florence & 987354 & 366039 & 8,1 & 280,9 & 124 \\
\hline Gênes & 851283 & 582320 & 9,1 & 463 & 111 \\
\hline Reggio diabre & 550323 & 180686 & 20,5 & 172,8 & 62 \\
\hline
\end{tabular}

Si l'idée de «ville compacte » et donc de densité, constitue en soi un critère positif pour une métropole, Naples défraye surtout la chronique nationale et européenne plutôt par ses « échecs » : la désormais fameuse crise des déchets, la criminalité, le persistant retard économique et l'importance du travail informel (Manceau, 2011 ; Froment, 2006). Elle est ainsi classée avec constance parmi les métropoles européennes en retard de développement, dont le PIB par habitant est inférieur à $75 \%$ de la moyenne 
communautaire. En 2010, celui-ci affichait un indice de 65 (UE=27), en deçà des métropoles du nord comme Milan (179) ou Bologne (141).

En 2013, le chômage en région Campanie concernait 21,5\% de la population active contre 12,2\% pour l'Italie (Istat, 2013). Ce taux reste élevé et concentré dans la métropole napolitaine. Pour la même période, la province de Naples affichait un taux de 25,7\% de chômeurs. Facteur aggravant, si la jeunesse de la population représente un atout pour une métropole, à Naples, le taux de chômage des 15-24 ans a dépassé les 50\% en 2013 (Istat, 2013). La difficulté de trouver un emploi pour les jeunes issus du Mezzogiorno se caractérise notamment par un départ vers les régions du Nord de l'Italie et l'étranger (Bianchi, Provenzano, 2010). Dans ce contexte, l'opportunité offerte d'une réussite que pourrait générer le projet de Bagnoli en termes d'emploi est à interroger bien qu'elle reste difficile à estimer ${ }^{5}$.

Pour Peppe Lanzetta, écrivain, Naples concentre « tous les vices d'une mégapole sans en avoir la richesse » (Bozonnet, 2008) et plus que jamais l'agacement de l'Italie du nord pour financer un tonneau des danaïdes. Cet agacement se traduit en particulier par le sempiternel débat autour $d u$ " coût du Mezzogiorno » pour les régions du Nord. Il a constitué d'ailleurs un enjeu politique et électoral chers à un parti comme la Ligue du Nord à la fin des années 2000 qui jugeait que « l'Italie du Nord, riche et industrialisée, serait retardée dans son développement économique par le Sud, plus pauvre et par un État fiscalement rapace, corrompu et inefficace » (Machiavelli, 2001, p.130).

Document 1. Le site industriel de Bagnoli : 1904-1993.

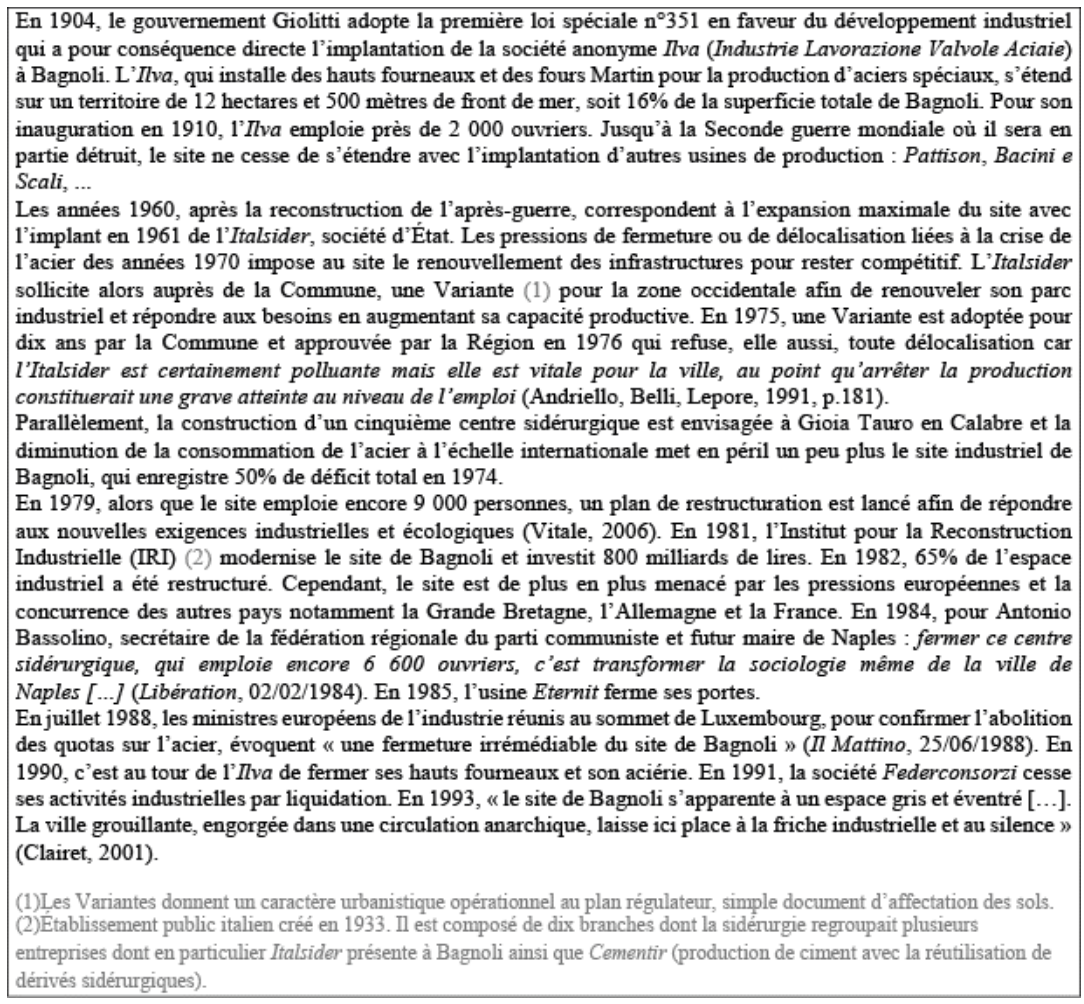

Auteur : Manceau, 2011.

Mais Naples a été et reste le principal centre industriel du Sud ${ }^{6}$ même si le processus de désindustrialisation opéré depuis les années 1980 a eu un impact fort sur le territoire en 
termes d'emploi et sur le paysage comme en témoigne l'ex-aire industrielle de Bagnoli (document 1).

13 Jusqu'à la fin des années 1960, l'essentiel de l'industrie du Mezzogiorno s'est localisé à Naples. « Géographiquement, les installations ont cerné la ville » (Vallat, 1998, p.202). Au cœur de Naples, et cette géographie est essentielle, ce sont deux grandes friches historiques - une dans la partie occidentale : Bagnoli, l'autre dans la partie orientale - qui font aujourd'hui l'objet d'un processus de transformation, soit près de 500 hectares.

\section{Le projet de reconversion du site de Bagnoli. Du gigantisme industriel au poumon « noir » de Naples}

14 Après sa fermeture en 1993 (document 1), les acteurs territoriaux amorcent le processus de transformation du site dont le coût total est estimé à 1220 millions d'euros (RodriguesMalta, 2006, p. 30). Ce processus s'articule autour de deux grandes étapes : la bonification des sols - toujours en cours - et la requalification du site, lancée en 2006. Bien qu'il soit inscrit dans un patrimoine paysager remarquable, celui d'un « amphithéâtre de collines volcaniques : les Champs Phlégréens et la colline de Pausilippe » (Mautone, 1978), le site de Bagnoli rencontre un fort problème d'image. Parmi les critiques les plus acerbes, Claudio Velardi, journaliste au quotidien méridional Corriere del Mezzogiorno qualifie le site de « désastre [...] le véritable trou noir de ces quinze dernières années à Naples » (Corriere del Mezzogiorno, 03/03/2009). Les récents évènements (incendie du musée, affaire de pollution aggravée, faillite de la société de transformation ...) n'ont fait que renforcer ce constat.

15 Le projet de transformation n'est lui-même pas épargné. En novembre 2008, on pouvait lire dans un article de La Repubblica d'Ottavio Lucarelli, les propos suivants : « le dessin de la nouvelle Bagnoli n'est ni de chair, ni de poisson. Il n'y a aucune identité définitive. Il y a plutôt, un projet dans lequel on a mis un peu de tout. Il ne semble pas y avoir une articulation cohérente avec une claire identité touristique » (La Repubblica, 04/11/2008).

16 Les travaux de transformation urbaine ont débuté en 2006 même si quelques projets sont antérieurs, comme la Città della Scienza qui a ouvert ses portes dès 2001. La trame générale vise à pérenniser «le caractère industriel des lieux» (Comune di Napoli, 2005). C'est d'autant plus important qu'au cours des années 1970/1980, le site de Bagnoli est perçu comme un quartier "sans chômage et sans camorra » (Rivière, 2004). Encore au milieu des années 2000, Ugo Marani, alors membre du conseil d'administration de la société en charge de la transformation urbaine évoquait lui aussi « Bagnoli comme une culture sans camorra, sans malavita, Bagnoli, c'était un rêve » (Entretien du 12/02/2007).

Le démantèlement de l'unité de production sidérurgique a donc été accompagné par une mission d'inventaire d'archéologie industrielle avec la sélection des éléments majeurs (haut-fourneau, entrepôts, ponton) (Rodrigues-Malta, 2006) : alors qu'initialement son démantèlement était envisagé, les fondations de l'aciérie sont conservées et mises en valeur (document 2). En décembre 2005, l'appontement nord qui servait à l'accostage des bateaux a été transformé en 800 mètres de promenade avancée sur la mer (document 3). 
Document 2. L'aciérie de Bagnoli.

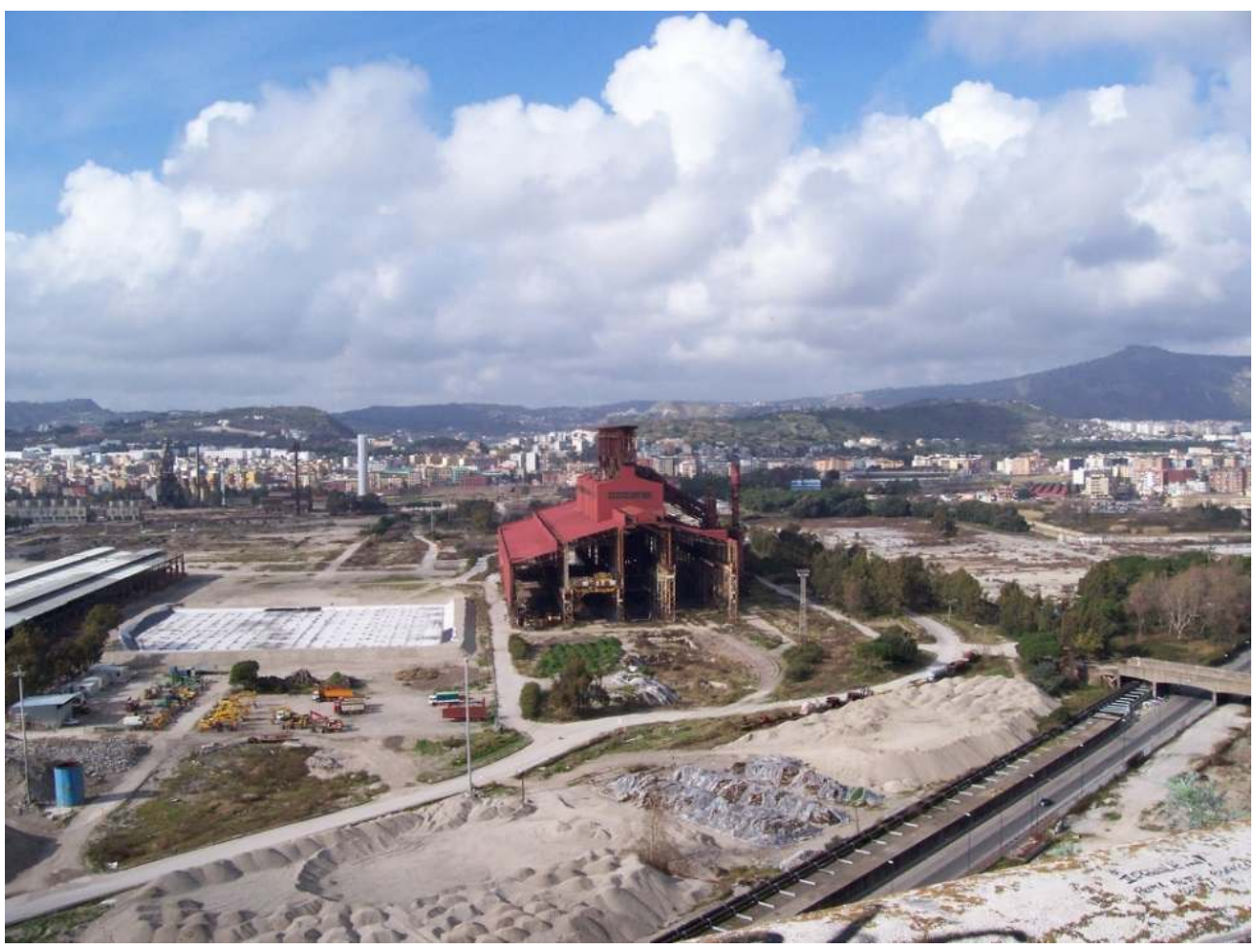

Manceau, 2007.

\section{Document 3. L'appontement nord de Bagnoli.}

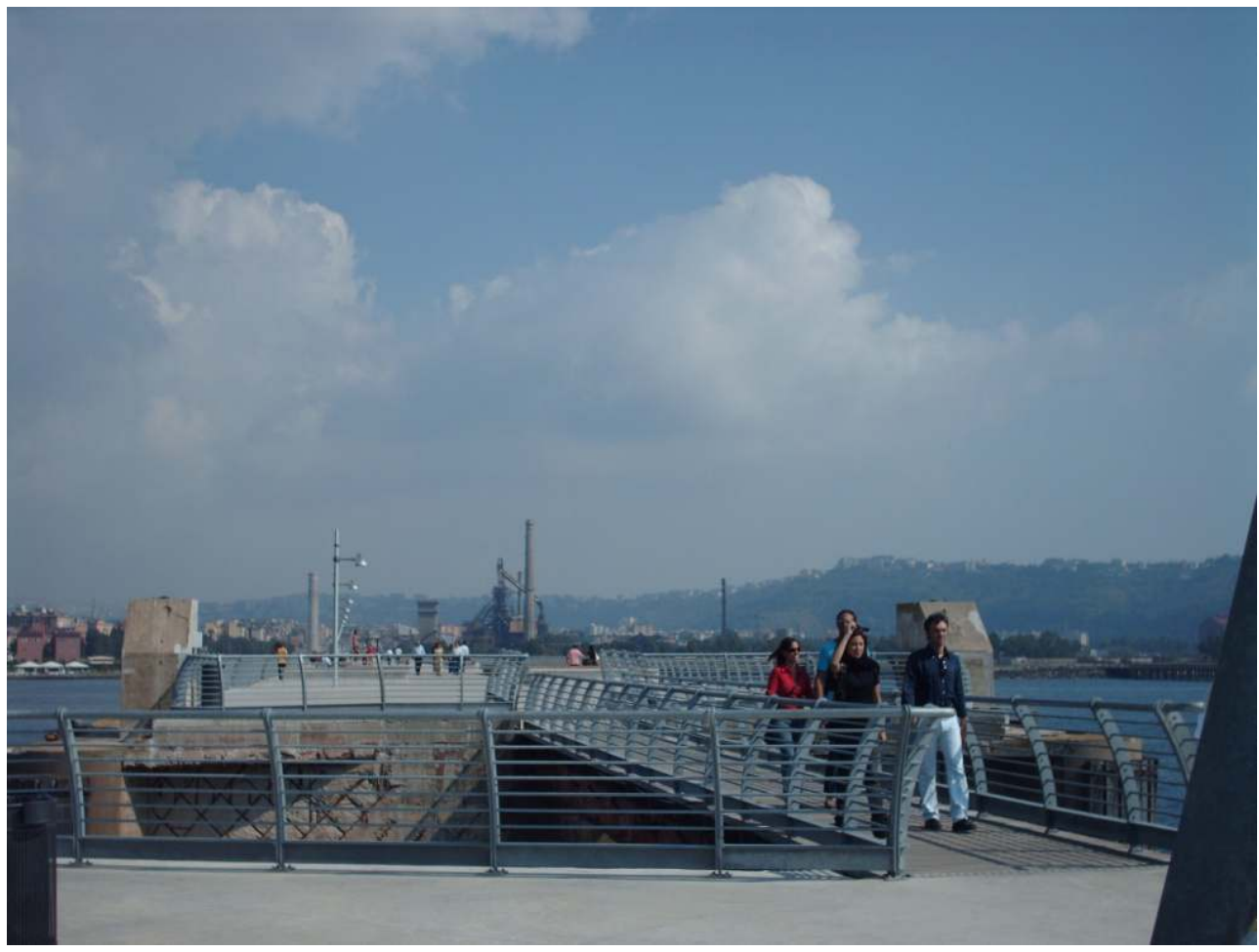

Manceau, 2006. 
Le centre multifonctionnel (la Porta del Parco) dont les travaux ont débuté en janvier 2007 a été inauguré en 2011 et accueille un auditorium, un centre de bien-être et une salle d'exposition (Figure 1).

Figure 1. Les projets de la transformation urbaine de Bagnoli.

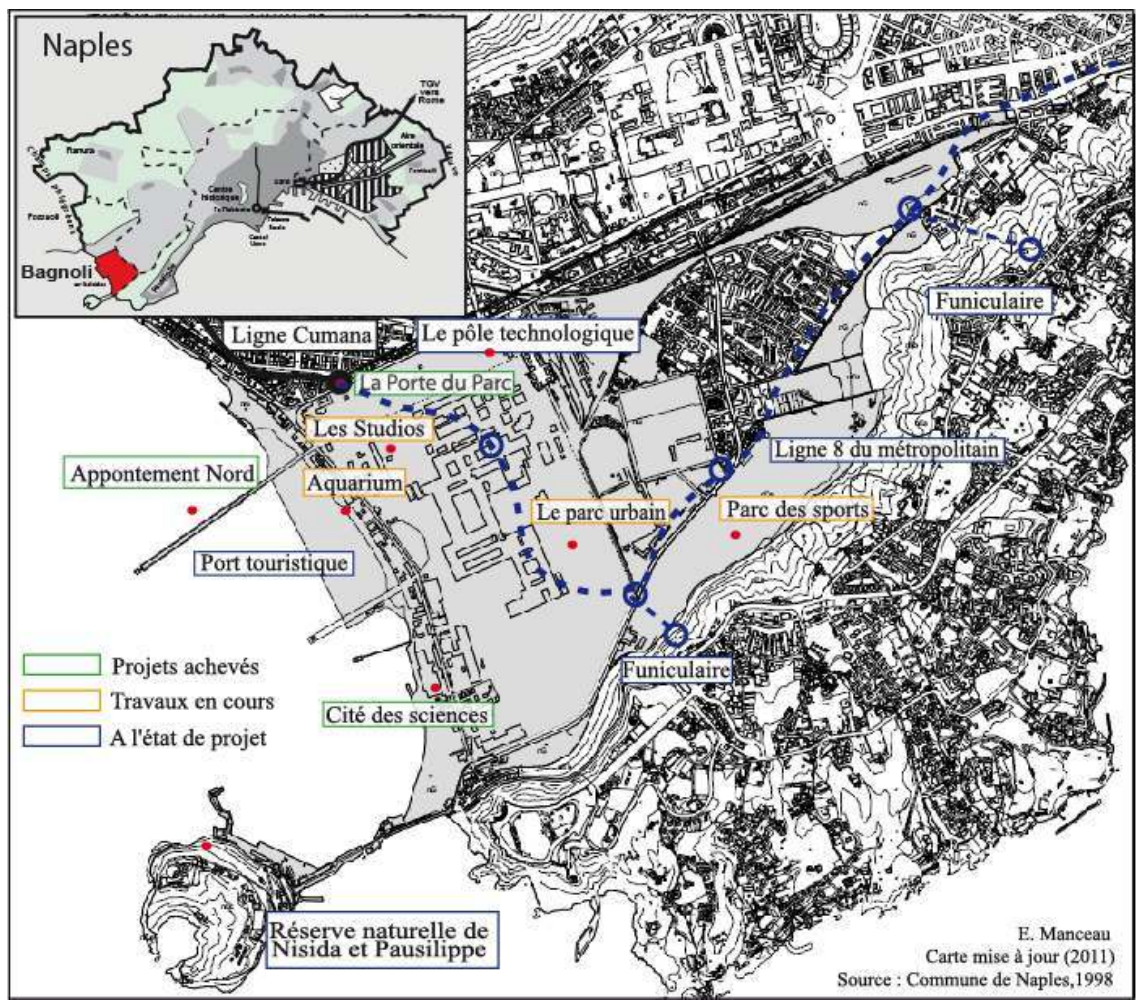

Plusieurs autres grands chantiers sont en cours (et parfois suspendus) parmi lesquels : le parc des sports qui s'étend sur 35 hectares. Il est pour le moment arrêté, confronté aux problèmes de pollution de ses sols. Après une visite de la Commission européenne en octobre 2013, la fin des travaux est prévue fin 2015. L'aquarium thématique - centre d'exposition de la mer - est achevé depuis 2011. Mais l'édifice a fait l'objet d'actes de vandalisme qui n'ont pas permis son ouverture.

20 Prévu dès les années 1990, le parc urbain qui devait être «le poumon vert de la ville», constitue le principal point noir de la «reconquête» en raison des travaux d'assainissement inachevés. Le parc doit s'étendre sur 160 hectares dont 33 hectares de plages. Des modes de circulation douces sont prévus avec $8,5 \mathrm{Km}$ de pistes cyclable. En matière d'infrastructures, les travaux de la future Ligne 8 de métro sont encore à l'état de projet comme le pôle technologique et le port touristique. Pour les Studios de Naples (production audiovisuelle et multimédia), seulement 15\% du projet était réalisé en 2014 (BagnoliFutura, 2014).

21 L'état des lieux autour de la reconversion du site de Bagnoli, symbole identitaire national et local fort de l'histoire industrielle au début des années 1990, offre aujourd'hui la réalité d'un entre deux au sort incertain caractérisé par la difficulté de faire aboutir le processus de transformation, une gouvernance souvent qualifiée d' «inefficace », de corrompue et épinglée par les instances européennes (Manceau, 2011 ; Rivière, 2011). 


\section{Le projet de Bagnoli : d'abord la reconquête de la ville}

La réhabilitation des friches portuaires et industrielles s'inscrit aujourd'hui dans la « circulation mondiale des modèles urbanistiques des waterfront » (Rivière, 2011). Elle est une composante de la compétitivité et du rayonnement des villes au même titre que d'autres grands projets d'infrastructures et d'équipements culturels (gare TGV, aéroport international, parcs à thèmes) et appréhendée comme telle par les territoires. Le territoire napolitain cherche à participer à ce mouvement plus général mais les projets portés, à l'exemple de la reconversion de Bagnoli, ne permettent pas à une échelle plus fine de « recoudre » le tissu urbain ${ }^{7}$.

\section{La transformation du site de Bagnoli : «toutes les fées sur le berceau »}

Partout en Europe, la réhabilitation des sites industriels est une opportunité pour répondre aux défis relevés par l'étalement urbain, le renforcement des disparités territoriales et permettre une "croissance intelligente, durable et inclusive " (SDEC, 1999 ; Agenda Territorial, 2007 ; Stratégie Europe 20/20, 2010). Elle est d'ailleurs portée symboliquement et cofinancée par l'Union européenne à travers sa politique de cohésion qui vise à réduire l'écart entre les niveaux de développement des territoires. Avec deux tiers de la population européenne vivant aujourd'hui en zone urbaine, la ville constitue le niveau d'intervention privilégié de l'Europe (Rapport Perben, 2008; Union européenne, 2011). Mais derrière ces discours répétés, ces injonctions plus ou moins itératives pour obtenir des financements, que signifient sur le terrain localement à Naples ces intentions?

Les acteurs institutionnels locaux, régionaux et nationaux s'accordent unanimement sur ces enjeux identifiés par la Commission. Cette convergence des orientations - qui vise aussi à capter les fonds communautaires - se lit dans les plans de planification et de programmation, principaux vecteurs de la volonté politique pour la transformation du site de Bagnoli (Figure 2).

25 A l'échelle communale, le plan urbanistique de Naples prévoit la récupération du site de Bagnoli comme une réponse à l'étalement urbain, à la sauvegarde des espaces verts (PUC di Napoli, 2004).

A l'échelle provinciale, qui correspond aujourd'hui à la Città metropolitana au sens institutionnel italien ${ }^{8}$, le Plan Territorial de Coordination (PTC), « conformément à la «vision» de la Région à travers son Plan Territorial Régional, vise une réorganisation polycentrique du territoire afin d'organiser le système métropolitain caractérisé par la fonction d'attraction de la capitale et certaines zones avec des niveaux élevés de spécialisation [...]. Parmi les nouvelles centralités du territoire se trouve Bagnoli dont les interventions visent un rôle de "charnière territoriale» entre la capitale (Naples) et le reste du territoire provincial » (Provincia di Napoli, 2008, pp.63-64).

La volonté affichée pour Naples et son échelle métropolitaine est à la fois de créer une nouvelle centralité caractérisée par de nouvelles fonctions culturelles et touristiques mais aussi de constituer une vitrine et lui permettre de s'affirmer à l'échelle nationale comme internationale: R. Rodrigues-Malta témoignait à propos de la transformation de 
l'interface urbano-portuaire dans les années 1990 qu'il s'agissait d'accessoires pour « look normal », d' " ustensiles " pour aider le retour à la normalité citadine, à la quotidienneté urbaine » (Rodrigues-Malta, 2001) et contrebalancer les représentations négatives.

Figure 2. Articulation des documents de programmation et de planification liée à la transformation urbaine de Bagnoli.

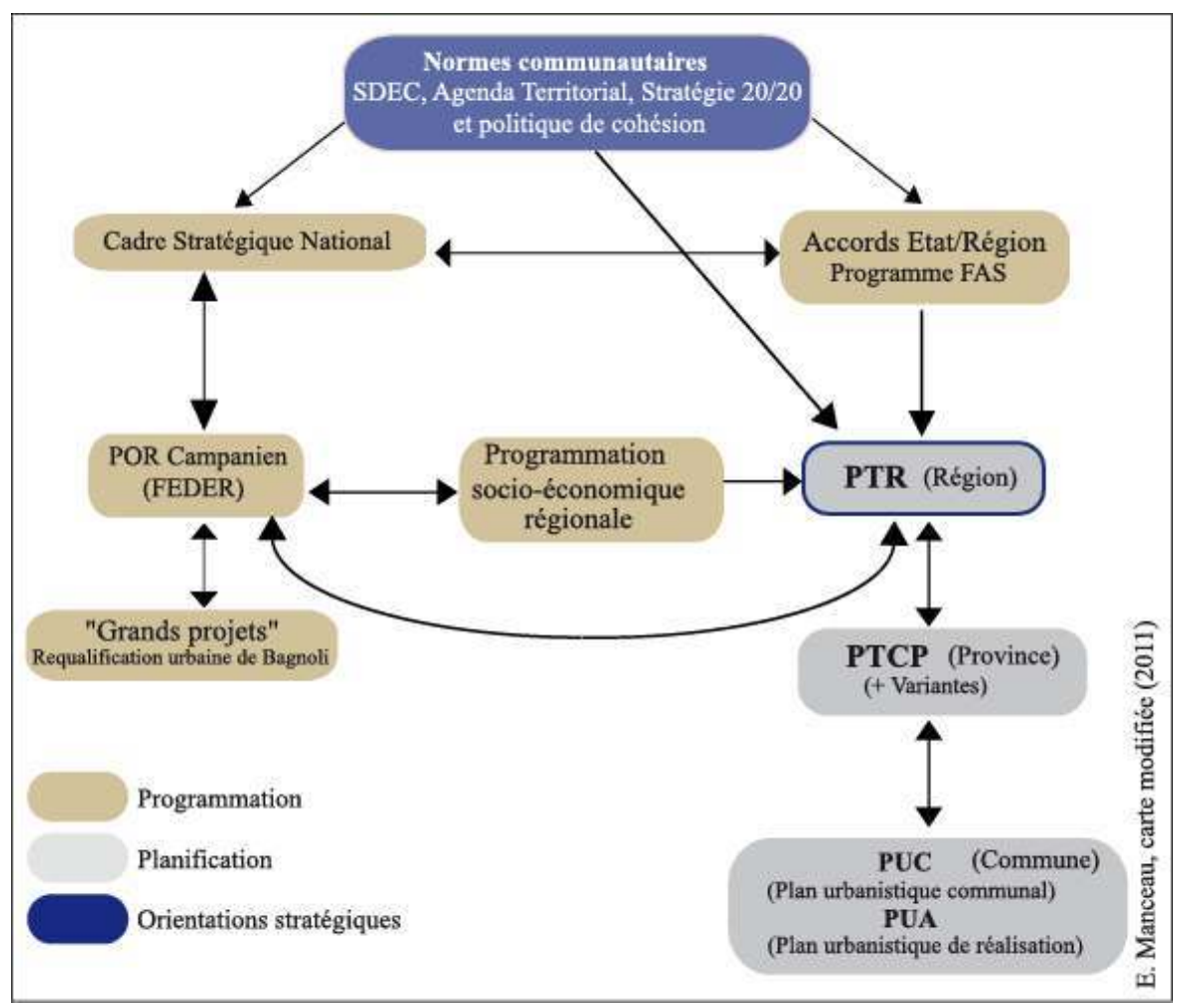

A l'échelle régionale, le Plan Territorial Régional adopté en 2007 prévoit, à travers la requalification du site et plus largement les anciens espaces industriels, de s'inscrire dans une démarche de développement durable et « d'amélioration de la compétitivité des villes et les territoires concernés » (Regione Campania, 2007). Ce lien entre le niveau européen et la région n'a rien d'étonnant. Simonetta Volpe et Domenico Smarazzo (co-auteurs du PTR) rappellent que la rédaction du PTR « repose essentiellement sur les documents stratégiques de l'Europe [...]» (S. Volpe et D. Smarazzo, entretien 01/2007).

A l'échelle nationale et imbriqué au Programme des fonds structurels européens, un accord de programme cadre - l'équivalent des CPER français - a été adopté en 2007 entre l'État et la Région Campanie pour la dépollution du site. Mais au-delà de la participation à cette opération environnementale, le lien de l'Etat avec Bagnoli est plus complexe et ancien. En effet, l'Etat italien a toujours décidé du sort de Bagnoli. En 1904, la loi sur le développement industriel du Sud industrialise le site (document 1). Il préconise ensuite la fermeture du site dès la fin des années 1970 pour enfin lancer le processus de régénération urbaine en 1995 en accord avec Antonio Bassolino, maire de Naples. Au cours de l'été 2014, Matteo Renzi, Président du Conseil italien met sous tutelle la reconversion et la bonification du site témoignant que «si Bagnoli redémarre, le Sud redémarrera " coupant dans le même temps les propos du Maire de Naples, Luigi de Magistris ${ }^{9}$ sur le fait qu'il n'y aurait pas de commissaire extraordinaire (La Repubblica, 30/08/2014). Cela laisse par ailleurs entrevoir les rapports tendus entre l'Etat et la Commune de Naples. 
30 Enfin, à l'échelle européenne, la requalification de Bagnoli est inscrite dans les Programmes opérationnels des fonds structurels campaniens depuis $2000^{10}$ avec deux grandes phases qui s'imbriquent dans les programmes nationaux et régionaux : la première liée à la bonification et la seconde propre à la transformation.

31 En première analyse, les documents de programmation et de planification des différentes échelles institutionnelles semblent s'accorder parfaitement sur les enjeux territoriaux à relever Ils semblent être unanimes sur l'importance de requalifier le site de Bagnoli et le rôle de ce territoire pour "recoudre " le tissu urbain et pour la "fabrique " de la métropole. Encore que suivant les différents intervenants, les priorités ne sont pas de même nature et que les attentes, au-delà d'accords généraux et formels peuvent signifier des politiques de types différents sur des parties d'espace variés : refaire la centralité, un exemple de développement durable privilégié à Bagnoli, pour les Napolitains ou pour les investisseurs et les touristes internationaux.

\section{Les limites du projet : enchevêtrement politico-institutionnel, absence de stratégie territoriale et gouvernance impossible}

32 Si cet emboitement vertical des plans d'aménagement montre une vision presque idéale autour du projet de reconversion du site, en ce qui concerne la mise en œuvre, les modalités d'utilisation des crédits, les cohérences dans l'articulation des projets, la vision dite idéale devient rapidement un lieu d'affrontements, une somme d'occasions perdues. Bagnoli alors témoigne de l'anatomie d'un mode de fonctionnement de Naples et le début d'une série d'échecs annoncés, une absence de véritable stratégie territoriale et une gouvernance inefficace et impossible.

$\mathrm{Du}$ point de vue opérationnel, le premier plan de bonification environnementale est approuvé par l'Etat en 1994. Mais les travaux d'assainissement des sols ne débutent qu'en 1996 lorsque la Commune de Naples approuve le document opérationnel (variante occidentale). Toutefois, ce document ne propose que des orientations générales articulées autour de la sauvegarde de l'environnement naturel, de l'architecture industrielle et de l'implantation d'un grand parc urbain sans plus de précisions. Affiliée à ce plan, la transformation est donc subordonnée à la rédaction d'un Plan urbanistique exécutif, soumis en 1998 par la Commune de Naples. Ce plan est chargé de lancer la seconde phase de la requalification du site par l'implantation d'activités et d'infrastructures, des fonctions qualifiées d'« hautement représentatives du renouveau de Naples » (PUE, 2005, p. 2).

Bagnoli témoigne d'une ambition en elle-même dans le droit fil de la renaissance urbanistique de Naples des années 1990 mais qui va se retourner contre elle. Rançon peut-être de ces ambitions, mais aussi de la fixation des conflits institutionnels et politiques sur ce sujet, lui-même traduisant des oppositions plus profondes et plus « traditionnelles » dans la culture politico-institutionnelle de Naples, le Plan n'est adopté que sept ans plus tard, en mai 2005, allongeant le calendrier initial ${ }^{11}$.

Ce retard s'explique par plusieurs raisons politico-institutionnelles et opérationnelles qui permettent de mettre aussi en exergue les différents niveaux d'intervention - parmi lesquelles : 


\section{A l'échelle locale :}

- En 2001, pour des raisons de coût et de temps, l'opposition au Conseil Municipal conteste l'adoption du plan qu'elle considère comme mal ficelé. Elle souhaite connaitre en détail le Plan d'exécution en particulier la destination des sols afin de savoir comment les dépolluer (en profondeur ou seulement en surface)

- La composition de la Société de transformation urbaine est contestée, remise en cause par l'opposition qui critique la présence uniquement d'institutions bancaires (Lepore, 2007).

\section{Aux échelles locale/nationale :}

- La gestion du site connaît aussi des complications. Les sols appartenaient en partie à l' Italsider, société sidérurgique italienne, placée sous le contrôle de l'Institut de reconstruction industrielle. Il faudra deux années (1996) à l'IRI pour constituer la société en charge de la bonification : la Société Bagnoli S.p.A. En 2001, quand la Commune acquiert les terrains de l' Italsider, elle reçoit un lourd héritage de la société d'État Bagnoli Spa: les opérations de dépollution des sols n'ont pas été achevées.

\section{A l'échelle nationale/internationale :}

- Le changement de gouvernement national puis le retour de l'Etat sur le projet de bonification des sols qu'il considère incomplet en particulier sur la récupération environnementale et sur le déblaiement des déchets bloque financièrement le processus, phénomène ici plus classique et plus habituel dès lors que l'on veut, légitimement tenir compte des impératifs du développement durable.

- La candidature de Naples à l'America's Cup en 2003 va définitivement ralentir l'adoption du Plan d'exécution, alors qu'elle voulait témoigner de la puissance et de la géographie particulièrement accueillante pour cette manifestation à haut niveau de médiatisation. Les débats entre la Municipalité, la Région et l'État avaient étonnamment porté sur la réorientation du Plan pour répondre aux exigences de l'America's Cup.

Pour certains comme Pecoraro Scanio, président des Verts, cet évènement (l'America's Cup ) n'est encore qu'un « instrument pour débattre du sort de la baie de Naples » (Lepore, 2007), le même qui en 2008, alors Ministre de l'Environnement avait refusé une solution pour la crise des déchets à Naples. La candidature n'a à la fin pas été retenue mais elle a témoigné des attentes différentes des acteurs.

L'adoption du plan en 2005 pouvait laisser supposer que le projet allait enfin pouvoir avancer mais la fin de l'année 2009 et le premier semestre 2010 se sont annoncés particulièrement tumultueux. Le rapport de la Cour des Comptes italienne sur le : «plan d'achèvement de l'assainissement et de la requalification environnementale de l'ancien site industriel de Bagnoli-Coroglio » publié en novembre 2009, met en évidence plusieurs carences. Selon le rapport, « les mécanismes de réalisation sont inadéquats par rapport aux différents niveaux institutionnels et les travaux d'assainissements sont bloqués en raison de l'insuffisance de décharges disponibles pour le stockage des déchets ». L'une des conséquences directes de ce rapport est la visite d'euro-parlementaires le 4 mars 2010 suivie de plusieurs questions écrites au Parlement européen parmi lesquelles celles de Luigi de Magistris (ALDE) - maire de Naples depuis 2011. Le 17 mars 2010, il évoque le "gaspillage important de fonds communautaires pour la requalification de l'ancien site industriel de la zone de Bagnoli ». Le 3 juin 2010, Luigi de Magistris interroge de nouveau le Parlement à propos cette fois-ci des violations avérées des normes en matière de marchés publics sur le site de Bagnoli. Ces évènements associés à l'éviction d'Antonio Bassolino $^{12}$, au profit de Stefano Caldoro issu de la droite, aux élections régionales 
achèvent de relancer la polémique sur les quinze années de l'ère Bassolino, l'importance des fonds perdus et selon la presse comme pour certains élus : l' " absence de résultats visibles». À son arrivée, la nouvelle administration régionale évoquait même la possibilité de révoquer les fonds pour Bagnoli.

Tableau 2. Atouts, opportunités, faiblesses et menaces du site de Bagnoli.

\begin{tabular}{|c|c|c|}
\hline Atouts & Faiblesses & \\
\hline $\begin{array}{l}\text { - Situation géographique } \\
\text { - Paysage des Champs Flégréens } \\
\text { - Cité des Sciences } \\
\text { - Plan urbanistique executif de } \\
\text { Bagnoli-Coroglio }\end{array}$ & $\begin{array}{l}\text { - Bureaucratie } \\
\text { - Pollution des sols } \\
\text { - Perception d'un quartier sans } \\
\text { opportunités } \\
\text { - Perte d'identité territoriale } \\
\text { - Manque d'attractivité du territoire }\end{array}$ & \\
\hline Opportunités & Menaces & \\
\hline $\begin{array}{l}\text { - Città metropolitana } \\
\text { - Organisation d'évènements } \\
\text { (America's Cup,...) }\end{array}$ & $\begin{array}{l}\text { - Conflits politico-institutionnels } \\
\text { - Calendrier électoral } \\
\text { - Images négatives } \\
\text { - Criminalité } \\
\text { - Métropolisation par l'accroissement } \\
\text { de la compétitivité entre les villes }\end{array}$ & 蒈 \\
\hline
\end{tabular}

L'absence de "vision » stratégique du Plan de reconquête du site, les nombreux conflits politico-institutionnels entre l'État, la Région et la Commune de Naples d'un côté, et au sein même de la Municipalité de Naples entre la majorité et l'opposition de l'autre, la désorganisation institutionnelle autour de la candidature ratée à l'America's Cup en 2003, le calendrier électoral ou encore les faits de corruption (Tableau 2) permettent de comprendre les raisons de l'échec de la reconversion du site de Bagnoli.

\section{La Città metropolitana : un gage de coordination pour la reconversion de Bagnoli?}

Si l'objectif principal de la reconquête du site de Bagnoli pourrait permettre sur le plan fonctionnel la transition métropolitaine de Naples à travers la création d'une nouvelle centralité, la transformation pourrait aussi sur le plan institutionnel fédérer les acteurs locaux et permettre l'affirmation de la toute récente Città metropolitana ${ }^{13}$ de Naples.

En effet, la Città metropolitana constitue un nouveau périmètre institutionnel depuis le $1^{\mathrm{er}}$ janvier 2014 censé remplacer la province et gérer la ville à une échelle plus vaste que celle de la Commune. Sur le papier, ses compétences portent sur la programmation, la planification, la coordination et la gestion intégrée des services, des infrastructures et des réseaux de communication (Camera dei Deputati, 2013).

De ce point de vue, la reconquête de Bagnoli pourrait pleinement s'inscrire dans le périmètre d'intervention de la Città metropolitana. En effet, la Province de Naples participe au consortium de la société de transformation BagnoliFutura. Certes actionnaire minoritaire de la société, discrète, voire «inexistante» dans le processus de transformation, jusqu'à la fin des années 2000, elle attribue une place croissante au projet de reconversion du site au sein de sa proposition de Plan Territorial de Coordination en 2008. On pourrait supposer que cette place est liée à l'institutionnalisation de la Città 
metropolitana. En effet, la proposition de Plan intervient un an avant la loi de 2009 sur l'autonomie fiscale. Mais voilà, la question métropolitaine vient bouleverser les rapports institutionnels et comme l'évoquait déjà $P$. Coppola à propos du processus de régionalisation, «[...] tout s'agite et quelque chose de neuf est en train de naitre, mais les niveaux effectifs de l'action souffrent de ce désordre créatif [...] (Coppola, 2003, p.76). Et ces «niveaux effectifs de l'action» que sont les échelles locale et régionale réduisent comme peau de chagrin les chances de la Città metropolitana de jouer sur l'échiquier de la reconquête de Bagnoli.

A l'échelle locale, Bagnoli est située entièrement sur la commune de Naples, qui disposait avant la faillite de l'entreprise de $90 \%$ des parts la société de transformation. Les récents échanges entre M. Renzi et L. de Magistris à propos de la nomination d'un commissaire extraordinaire pour la reconversion du site posent le décor. L'opération de reconversion reste avant tout une chasse gardée de la commune même si le site est bel et bien sous tutelle.

A l'échelle régionale, Antonio Bassolino, gouverneur de la Région Campanie depuis 2001, adopte en 2004 la loi sur le «Gouvernement du territoire $»^{14}$. Cette loi prévoit que la "planification territoriale en cascade fait référence seulement aux trois niveaux de collectivités territoriales traditionnelles : la région, la province et la commune [...] dans ce contexte, la région garde le contrôle sur les politiques locales stratégiques. Cette difficulté à déterminer une institution métropolitaine redonne plus de force politicoinstitutionnelle à la région qui se charge de plus en plus des dossiers d'envergure métropolitaine et intercommunale » (Di Ciommo, 2006, p.12). La reconversion de Bagnoli si chère à Antonio Bassolino au milieu des années 1990 constitue-t-elle alors un « dossier d'envergure » qui doit être géré par le niveau régional comme peut l'être par exemple le système de transport régional?

\section{Conclusion}

Une première lecture "banale ", fondée essentiellement à partir des documents, des projets dits stratégiques qui se révèlent régulièrement sérieux, cohérents et qui déclinent avec beaucoup de précisions, schémas, cartes, opérations, emboitements des espaces, montages d'opérations et programmes temporels d'obtention et d'engagement des financements, montre que Bagnoli présente de nombreux caractères de modèle idéal. Classiquement encore, on sait que ces montages se déroulent dans le temps et que les thèmes porteurs, les objectifs généraux, les références en matière de développement changent avec le temps, les porteurs de projets, les environnements plus ou moins contraints par les financements et les impératifs de rationalisation et de restriction budgétaires. Alors Bagnoli n'a rien de bien différent des contrats de plan Etat-Régions en France ou des politiques des fonds européens. Il faut tenir compte des impératifs en matière de développement durable mais aussi tenir compte des effets de mode, le tourisme international, la volonté de constituer ou de renforcer les clusters ou les districts italiens, profiter des opportunités foncières pour offrir des "poumons verts", restructurer la ville sur la ville.

48 La réhabilitation de friches industrielles qui plus est situées quasiment au centre de la ville relève très simplement du travail de Sisyphe. Ici, comment concilier la volonté de maintenir une symbolique industrielle et faire de Naples une ville moderne? Cette lecture conforte l'attention qu'il faut accorder aux détails, aux opérations quotidiennes, à 
la gestion et à l'évaluation précise et suivie du déroulement et des bifurcations certaines imposées par l'extérieur, d'autres venant de la réalité locale et de ses vicissitudes. Elle montre aussi tout l'intérêt mais aussi les limites de travaux ne reposant que sur des textes et des programmes dont les discours sont globalement bien calés sur les attentes des acteurs politiques et sur celles des financiers.

La seconde lecture s'éloigne des remarques précédentes d'ordre général et répétitif. Il s'agit de Bagnoli dans Naples, il est question de la métropole napolitaine, de ses modes de fonctionnement, de ses logiques de gouvernance, de répartition des tâches et des enveloppes. On est bien encore au cœur de l'Italie du sud, de ses ancrages comportementaux, de son clientélisme acharné et de ses modes de régulation singuliers où la criminalité, la corruption vont bien au-delà des enjeux et des modalités habituels du jeu des rapports de force. Et Bagnoli, alors, c'est la face visible, émergée, d'un continent napolitain où jouent, sans jeu de mots, en permanence, les forces volcaniques du magma. C'est en quelque sorte l'irruption apparente, révélée d'une société locale qui sait construire des projets tirés au cordeau, qui est championne dans l'art de trouver des fonds, avec sans doute la bénédiction ou l'ignorance tacite des gouvernements de Rome ; Bagnoli, c'est l'irruption excessive de phénomènes habituels, à un effet de lisibilité renforcé comme l'a été la grève des ordures.

Enfin, un autre regard concerne la Città metropolitana et la fabrique urbaine, thème à la mode dans la littérature et chez les urbanistes. On en connait les bases; réinventer de l'urbanité, recoudre la ville et lier les périphéries, faire une ville durable, rendre public de nombreux espaces de fait privatisés, harmoniser les cultures, identitaires et les espaces de la plus haute modernité et de haute technologie et de forte médiatisation... Mais Bagnoli du moins à ce jour semble d'abord avoir à se transformer, à réaliser sa propre requalification avant même que de prétendre jouer un rôle moteur et réel dans cette Città metropolitana, encore moins dans les attentes plus ou moins rêvées de la métropolisation. Le nouveau statut de Città metropolitana semble, apparemment, modifier le paysage institutionnel et pourrait donner des gages de coordination et d'intégration plus affirmée. Il n'est pas certain qu'au fond cette évolution institutionnelle ne reste à nouveau que formelle: dans une bouteille nouvelle, on risque de faire comme avant le même vin: Cambiare tutto per non cambiare nulla!

BIBLIOGRAPHIE

ANDRIELLO V., BELLI A., LEPORE D. (1991), Il luogo e la fabbrica. L'impianto siderurgico di Bagnoli e l'espansione occidentale di Napoli, Napoli, Graphotonic.

BAGNASCO A. (1977), Tre Italie, la problematica territoriale dello sviluppo italiano, Bologna, Il Mulino. BOURDEAU-LEPAGE I., GASCHET F., LACOUR C., PUISSANT S. (2012), Métropolisation, le triomphe des processus agglomératifs, Regards sur la ville, Economica, pp. 7-33.

BOZONNET J-J. (2008), « Le désespoir et la honte d'être napolitain », Le Monde, 22 mai. 
CAMERA DEI DEPUTATI (2013), Città metropolitane, province, unioni e fusione di comuni, documentazione per l'esame di Progetti di legge, Roma.

CLAIRET S. (2001), « Entre mutations politiques et mutations urbaines, les représentations du projet urbain à Naples », Politiques urbaines à Naples et à Marseille, regards croisés, Méditerranée, 96, 1-2, pp.107-110.

COPPOLA P. (2003), Le processus de régionalisation dans l'expérience italienne, Territoire 2020, 8 , pp.75-79.

CORTE DEI CONTI (2009), Piano di completamento della bonifica e del recupero ambientale ex-sito industriale Bagnoli, Roma.

COURLET, C., PECQUEUR, B., (1991), Systèmes locaux d'entreprises et externalités : un essai de typologie, Revue d'Économie Régionale et Urbaine, 3/4, pp. 391-406.

DELPIROU A., RIVIERE D. (2013), « Réseaux urbain et métropolisation en Italie : héritages et dynamiques », Géoconfluences. Mis en ligne le 5 décembre 2013, http://geoconfluences.enslyon.fr/informations-scientifiques/dossiers-thematiques/de-villes-en-metropoles/articlesscientifiques/reseau-urbain-et-metropolisation-en-italie-heritages-et-dynamiques.

DI CIOMMO F. (2006), Métropolisation et planification urbaine dans une perspective de développement durable, Rapport intermédiaire du post-doctorat, Paris, CSTB.

FELKAI I. (1984), « Sidérurgie européenne : "plus que” 100000 emplois à laminer », Libération, 2 février.

FROMENT P. (2003), « Les espaces publics dans les politiques patrimoniales à Naples : Place du Plébiscite et Place Dante », Rives nord méditerranéennes, Patrimoine et politiques urbaines en Méditerranée, 16, pp. 99-118.

FROMENT P. (2006), « Districts, entreprises et travail au noir en Italie du Sud », Méditerranée, 106, pp.85-93.

FROMENT P., RIVIÈRE D., « Naples », Encyclopcedia Universalis [en ligne], consulté le 23 octobre 2014, http://www.universalis.fr/encyclopedie/naples/

FUCCILLO R., (2014), « Renzi: “Bagnoli riparte col commissario, con tempi certi e riparte pure il Sud" », La Repubblica, 30 août.

HALBERT L., CICILLE P., ROZENBLAT C. (2012), Quelles métropoles en Europe ? Des villes en réseau, Travaux de la DATAR n¹6, Paris, La Documentation Française.

IMPERIALI E. (1988), « La Cee è contro Bagnoli », Il Mattino, 25 juin.

LACOUR C., PUISSANT S. (1999), La métropolisation: croissance, diversité, fractures, Paris, Anthropos, Coll.Villes, pp. 63-113.

LEPORE D. (2007), « Il riuso dell'area di Bagnoli », in BELLI A., (a cura di), Non è così facile. Politiche urbane a Napoli a cavallo del secolo, Franco Angeli.

LUCARELLI O. (2008), « Velardi boccia Bagnoli: “Non ha identità turistica” », La Repubblica, 4 novembre.

MACHIAVELLI M. (2001), « La Ligue du Nord et l'invention du "Padan" », Critique internationale, 10, pp. 129-142.

MANCEAU E. (2011), La politique de cohésion européenne et l'aménagement du territoire en Italie. L'exemple de la région Campanie, Thèse de doctorat, Université Paris-Diderot, $507 \mathrm{p}$. 
MAUTONE M. (1978), « Un quartiere periferico della città di Napoli: Bagnoli », Annali della facoltà di lettere e filosofia dell'università di Napoli, pp. 445-472.

PARLEMENT EUROPÉEN (2010), Gaspillage important de fonds communautaires pour la requalification de l'ancien site industriel de la zone de Bagnoli, Questions parlementaires posée par Luigi de Magistris (ALDE) à la Commission, 17 mars.

PARLEMENT EUROPÉEN (2010), Violation avérée des normes en matière de marchés publics concernant l'ancien site industriel de Bagnoli, Question parlementaire posée par Luigi de Magistris (ALDE), 3 juin.

PERBEN D. (2008), Imaginer les métropoles d'avenir, Paris, Présidence de la République.

PROVENZANO G., BIANCHI L. (2010), Ma il cielo è sempre più su? L'emigrazione meridionale ai tempi di Termini Imerese, Roma, Castelvecchi.

REPUBBLICA ITALIANA, Legge 8 giugno 1990, n 142, Ordinamento delle autonomie locali, Aggiornato alla G.U del 15 giugno $1998, n^{\circ} 137$.

REPUBBLICA ITALIANA, Legge costituzionale $\mathrm{n}^{\circ} 3$ del 2001, Riforma del Titolo V della parte seconda della costituzione italiana (1948).

REPUBBLICA ITALIANA, Legge 5 maggio 2009, $\mathrm{n}^{\circ} 42$ in materia di federalismo fiscale nata dalla c.d. bozza Calderoli.

RIVIÈRE D. (2004), L'Italie, des régions à l'Europe, Paris, A. Colin.

RIVIÈRE D. (2011), « Le renouvellement urbain et la politique européenne de cohésion, de Naples à Saint-Denis ", Entre recherche et expertise : itinéraires en villes-ports. Hommage à Rachel RodriguesMalta, Rives méditerranéennes, pp. 103-123.

RODRIGUES-MALTA R. (2001), « Naples-Marseille : Waterfront attitude », Politiques urbaines à Naples et à Marseille : regards croisés, Méditerranée : Revue géographique des pays méditerranéens, 96, pp. 97-106.

RODRIGUES MALTA R. (2006), « La dimension physique et urbanistique de la régénération de l'interface ville-port », Working group SUDEST, Dimension physique et urbanistique de la régénération urbaine, Livorno.

ROZENBLAT C., PATRICIA C. (2003), Les villes européennes: analyse comparative, DATAR, Paris, La Documentation française.

SARACENO P. (1980), « Cattedrali nel deserto? », Nord e Sud, n 9.

TRIGILIA C. (1986), Grandi partiti e piccole imprese: comunisti e democristiani nelle regioni a economia diffusa, Il Mulino.

VALLAT C., MARIN B., BIONDI G. (1998), Naples : démythifier la ville, Paris, L’Harmattan.

VELARDI C. (2009), « Bagnoli, dopo 15 anni prendiamo atto del disastro », Corriere del Mezzogiorno, 3 mars 2009.

VITALE A. (2006), « Dismissione di aree siderurgiche: strategy, progetti e realizzazioni. Il caso della ex-Ilva a Napoli », Industrial heritage and urban transformation productive territories and industrial landscape, TICCIH, XIII International Congress, Terni-Roma, 13 p.

Documents stratégiques, de planification et de programmation

COMMISSION EUROPÉENNE (1999), Schéma du développement de l'espace communautaire (SDEC), Potsdam. 
COMMISSION EUROPENNE (2007), Agenda territorial de l'Union Européenne, Leipzig.

COMMISSION EUROPENNE (2010), Stratégie Europe 20/20, Bruxelles.

COMMISSION EUROPENNE (2011), Les villes de demain. Défis, visions et perspectives, Bruxelles.

COMUNE DI NAPOLI (1998), Variante di salvaguardia, Napoli.

COMUNE DI NAPOLI (2004), Piano regulatore generale (et Variantes générales au PRG), Napoli.

COMUNE DI NAPOLI (2005), Piano urbanistico attuativo di Coroglio-Bagnoli, Napoli.

PROVINCIA DI NAPOLI (2008), Proposta di Piano Territoriale Coordinamento Provinciale (PTCP), Napoli.

REGIONE CAMPANIA (2004), Programma Operativo Regionale 2000-2006 (POR), Testo aggiornato alla

Decisione della Commissione Europea del 15 dicembre 2004.

REGIONE CAMPANIA (2007), Programma operativo FESR 2007-2013.

REGIONE CAMPANIA (2007), Piano Territoriale Regionale (PTR).

\section{Site internet}

Société de transformation urbaine de Bagnoli : www.bagnolifutura.it

ISTAT: http://www.istat.it/it/

EUROSTAT: http://epp.eurostat.ec.europa.eu/portal/page/portal/eurostat/home/

\section{Entretiens}

Ugo Marani, Membre du Conseil d'administration de la Société BagnoliFutura, Février 2007.

Simonetta Volpe et Dominico Smarazzo, Région Campanie, Janvier 2007.

\section{NOTES}

1. L'enquête n'a pas permis pour le moment d'identifier les coupables et les raisons d'un tel acte. Néanmoins, de fortes suspicions portent sur la culpabilité de la Camorra.

2. Bagnolifutura est la société de transformation en charge de gérer la transformation urbaine du site industriel de Bagnoli. Les actionnaires sont la Commune de Naples (90\%), la Région Campanie (7,5\%) et la Province napolitaine (2,5\%).

3. Cet aspect ne sera pas abordé dans cet article.

4. Sur fond d'opération "Mains Propres »- qui révèle au grand jour le scandale des « pots-de-vin » dans le monde politique italien - la « renaissance napolitaine » renvoie à la politique menée par Antonio Bassolino, après son élection en 1993. Cette politique repose sur la réappropriation du patrimoine architectural et paysager par les Napolitains et sur plusieurs opérations phares de requalification: la relance des travaux du métropolitain, l'organisation chaque année du « Mai des monuments ", sorte de journée du patrimoine local élargie au mois de mai et le réaménagement de la Place du Plébiscite, restaurée en 1994 et interdite à la circulation. La place du Plébiscite constitue le symbole du renouveau napolitain en ce sens qu'elle caractérise la volonté d'agir sur les espaces les plus emblématiques du centre (Froment, 2003).

5. Au-delà de la difficulté d'évaluer le nombre d'emplois générés dans les phases " chantier » et exploitation du site, les suspensions à répétition des travaux du chantier liées aux différentes affaires rendent l'exercice encore plus complexe. 
6. Depuis la fin du $19^{\text {ème }}$ siècle, plusieurs phases d'industrialisation ont marqué le territoire napolitain, résultantes de politiques portées en faveur du développement industriel (loi spéciale de 1904 pour le développement industriel de Naples, loi de 1957 liée aux aires et noyaux de développement industriel, etc.). À partir des années 1970, s'opère un processus de desserrement industriel dans l'arrière-pays napolitain (Caserte, Averse, etc.). Ce phénomène touche plus largement l'ensemble du Mezzogiorno et aboutit à la naissance de " cathédrales dans le désert » (Bari, Brindisi, Tarente, Naples, etc.) (Saraceno, 1980). La province de Naples rassemble $45 \%$ de toute les entreprises publiques du Sud (FIAT, Italsider (sidérurgie), Finsider/Armco (laminés zingués), Alfa Sud) et 32\% des entreprises privées parmi lesquelles Olivetti et Remington (deux firmes américaines spécialisées dans le matériel de bureau), Mobil oil (pétrole), Philip's, Varta AG (accumulateur électrique) (Vallat, 1998).

7. Par exemple les travaux du projet de construction de gare nouvelle de la Ligne à Grande Vitesse située à Afragola au nord de Naples dont le coût avait été estimé à 77 millions d'euros ont été stoppés en 2012 en raison de la faillite de l'entreprise et de la présence de la Camorra dans les marchés publics. Ces travaux ont souvent été qualifiés

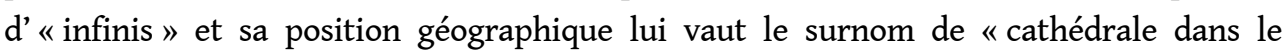
désert ».

8. Le $1^{\text {er }}$ janvier 2014, les Villes métropolitaines de Turin, Milan, Venise, Gènes, Bologne, Florence, Bari, Naples et Reggio de Calabre ont été instituées. Ces Villes métropolitaines se sont substituées aux Provinces, le $1^{\text {er }}$ juin 2014. Rome Capitale en raison de son statut spécial dispose d'un règlement spécifique. Le territoire de la Ville métropolitaine coïncide à celui de la Province. La Città metropolitana aura des compétences de programmation, planification, coordination et de gestion intégrée des services, des infrastructures et des réseaux de communication (Camera dei Deputati, 2013).

9. Luigi de Magistris a été suspendu de son mandat de maire de Naples en septembre et octobre 2014 en raison d'une enquête en cours pour abus de pouvoir dans le cas d'un procès pour écoute téléphonique sans autorisation quand il était magistrat. Le retour à son mandat de maire a été autorisé le 30 octobre 2014 par la cour administrative.

10. Le Programme opérationnel est un outil financier approuvé par la Commission. Il établit les lignes stratégiques, composées d'axes et de mesures, pour l'emploi des fonds structurels de l'Union Européenne à l'échelle de la région.

11. Par ricochet, ce retard a notamment engendré le blocage des crédits européens par la Commission.

12. Antonio Bassolino a été Président de la Région Campanie entre 2000 et 2010.

13. La création de la Città metropolitana est issue de la loi de décentralisation $n{ }^{\circ} 142$ de 1990 - considérée comme une pierre angulaire à la décentralisation de la politique d'aménagement du territoire - qui définit l'aire métropolitaine comme l'ensemble des « communes qui ont en commun des activités économiques, [...] des relations culturelles et des caractéristiques territoriales». Le statut de la Città metropolitana est ensuite renforcé par l'article 114 de la réforme constitutionnelle en 2001, qui affirme que la République est constituée des Communes, Provinces, Villes métropolitaines, Région et État. L'article 119, mis en œuvre par la loi de 2009, affirme de son côté l'autonomie fiscale de ces administrations.

14. Cette loi est issue de la réforme constitutionnelle de 2001 qui bascule un certain nombre de compétences aux régions. 


\section{RÉSUMÉS}

Dans un contexte de compétition accrue des territoires, c'est par le biais de projets d'aménagement d'envergure sur des espaces périurbains ou même centraux longtemps restés en marge que la plupart des grandes villes prétendant être ou devenir métropoles se positionnent pour s'affirmer sur le plan national, européen, voire international. Le site de Bagnoli, à Naples, à l'image des autres projets existants, concentre les composants nécessaires à un processus de transformation urbaine réussi. Pourtant, Bagnoli montre des limites qui peuvent remettre en cause l'opportunité offerte d'un projet structurant. Cet article présente ces limites qui se caractérisent par des rapports de force entre logiques territoriales nationales, régionales et locales, des images véhiculées négatives et l'absence d'une stratégie territoriale du projet.

In a context of territorial competition, most metropolitan cities wish obtain a national, European or international relevant position through majors urban projects in suburban and marginal areas. The case of Bagnoli, in the city of Naples, like others examples, includes some attributes in order to reach the target of a successful urban transformation process and the construction of the "Metropolitan Urban Factory". Nevertheless, in this case, urban policies are so confusing that it could undermine the opportunity of this project. This article investigates the relationships and links between actors at nationals, regional and local levels. It focuses on negative opinions and the lack of territorial strategy for this urban project.

Numerose città metropolitane desiderano affermarsi sul piano nazionale, europeo ed internazionale in un contesto di concorrenza territoriale attraverso progetti di riqualificazione urbana di aree un tempo periferiche e marginali. Il caso di Bagnoli, a Napoli, come altri progetti, concentra una serie di caratteristiche necessarie ad un processo di trasformazione urbana vincente, e può partecipare in questo senso alla "fabbrica" della città metropolitana. Nonostante ciò, questo progetto costituisce una forma di cacofonia delle politiche di pianificazione del territorio che può rimettere in questione l'opportunità offerta da un progetto strutturante. Questo articolo analizza in particolare i limiti definiti dai rapporti di forza tra logiche di tipo nazionale, regionale e locale, delle immagini negative trasmesse e della mancanza di una strategia territoriale forte attribuita al progetto.

\section{INDEX}

Mots-clés : friche industrielle, Città metropolitana, gouvernance territoriale, Naples, Bagnoli

Keywords : Industrial Wasteland, Metropolitan City, Territorial Governance

\section{AUTEUR}

\section{ELODIE MANCEAU}

UMR Géographie-cités, manceau.elodie@gmail.com 